\title{
Cytotoxic Withanolides from Tubocapsicum anomalum
}

Pei-Wen Hsieh,,$^{\dagger}$ Zih-You Huang, ${ }^{\dagger}$ Jyun-Hong Chen, ${ }^{\dagger}$ Fang-Rong Chang, ${ }^{\dagger}{ }^{*}$ Ching-Chung Wu, ${ }^{\dagger}$ Yu-Liang Yang, ${ }^{\dagger}$ Michael Y. Chiang, ${ }^{\ddagger}$ Ming-Hon Yen, ${ }^{\dagger}$ Shu-Li Chen, ${ }^{\dagger}$ Hsin-Fu Yen, ${ }^{\S}$ Tilo Lübken,,$^{\dagger}$ Wen-Chun Hung, ${ }^{\perp}$ and Yang-Chang $W u^{\dagger,}{ }^{\perp}, *$

Graduate Institute of Natural Products, School of Pharmcy, Kaohsiung Medical University, Kaohsiung 807, Taiwan; Department of Chemistry, National Sun Yat-Sen University, Kaohsiung 804, Taiwan; National Museum of Natural Science, Taichung, 404, Taiwan and National SunYat-Sen University-Kaohsiung Medical University Joint Research Center

${ }^{*}$ To whom correspondence should be addressed. For Y.-C. Wu: phone, (+886)-(0)7-312-1101 ext. 2197; fax: (+886)-(0)7-311-4773; e-mail: yachwu@kmu.edu.tw. For F.-R. Chang: phone, (+886)-(0)7-312-1101 ext. 2162; E-mail: aaronfrc@kmu.edu.tw.

${ }^{\dagger}$ Kaohsiung Medical University

National SunYat-Sen University

$\S^{\S}$ National Museum of Natural Science

${ }^{\perp}$ National SunYat-Sen University-Kaohsiung Medical University Joint Research Center 


\section{Table and Contents}

Table S1. The ${ }^{1} \mathrm{H}(400 \mathrm{MHz})$ NMR Data of Mosher's Esters of Compounds 1, 4, and 12 in

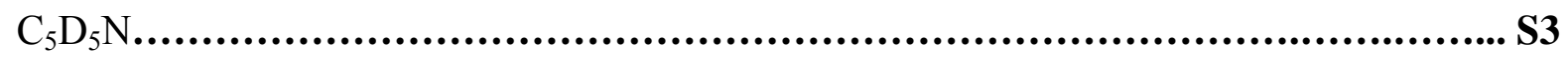

Table S2. ${ }^{13} \mathrm{C}$ NMR Data (100 MHz) of $\mathbf{1 8}-23$ in $\mathrm{C}_{5} \mathrm{D}_{5} \mathrm{~N}, \mathrm{CDCl}_{3}$, or $\mathrm{CD}_{3} \mathrm{OD} \ldots \ldots . . . . . .$. S4

Table S3. ${ }^{13} \mathrm{C}$ NMR Data $(100 \mathrm{MHz})$ of $24-27$ in $\mathrm{C}_{5} \mathrm{D}_{5} \mathrm{~N}$................................... S5

Table S4. ${ }^{1} \mathrm{H}$ NMR Data (400 MHz) of $\mathbf{1 8}-21$ in $\mathrm{CDCl}_{3}$, or $\mathrm{CD}_{3} \mathrm{OD} \ldots . . . . . . . . . . . . . . .$. S6

Table S5. ${ }^{1} \mathrm{H}$ NMR Data $(400 \mathrm{MHz})$ of 22 and 23 in $\mathrm{C}_{5} \mathrm{D}_{5} \mathrm{~N}$ or $\mathrm{CDCl}_{3} \ldots \ldots \ldots . . . . . . . . . . . . .$. S7

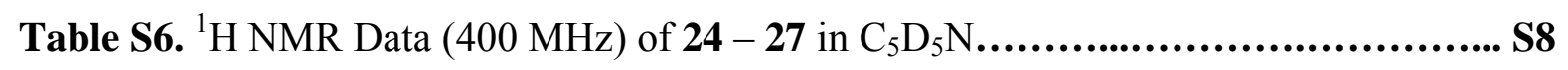

Figure S1. The Structures of Artificial Components (18-27) from Tubocapsicum anomalum....................................................................................S9

Additional Experimental Details. ................................................S10 
Table S1 (Supporting Information). The ${ }^{1} \mathrm{H}(400 \mathrm{MHz})$ NMR Data of Mosher's Esters of Compounds 1, 4, and 12 in $\mathrm{C}_{5} \mathrm{D}_{5} \mathrm{~N}$.

\begin{tabular}{|c|c|c|c|c|c|c|c|c|c|}
\hline \multirow[b]{2}{*}{ Position } & \multicolumn{3}{|c|}{1} & \multicolumn{3}{|c|}{4} & \multicolumn{3}{|c|}{12} \\
\hline & $S$-MTPA & $R$-МТРА & $\Delta\left(\delta_{S}-\delta_{R}\right)$ & $S$-МТРА & $R$-МТРА & $\Delta\left(\delta_{S^{-}} \delta_{R}\right)$ & $S$-MTPA & $R$-MTPA & $\Delta\left(\delta_{S}-\delta_{R}\right)$ \\
\hline 2 & 6.438 & 6.541 & -0.103 & 6.450 & 6.548 & -0.098 & 6.1875 & 6.2595 & -0.072 \\
\hline 3 & 7.252 & 7.305 & -0.053 & 7.210 & 7.264 & -0.054 & 6.4075 & 6.5425 & -0.135 \\
\hline 4 & 5.418 & 5.476 & -0.058 & 5.373 & 5.425 & -0.052 & 6.4200 & 6.4200 & +0.280 \\
\hline 6 & 3.672 & 3.615 & +0.057 & 3.477 & 3.426 & +0.051 & 4.5150 & 4.5150 & +0.123 \\
\hline \multirow[t]{2}{*}{7} & & & & 1.064 & 1.027 & +0.037 & & & \\
\hline & & & & 1.987 & 1.955 & +0.032 & & & \\
\hline 9 & & & & 0.940 & 0.918 & +0.022 & & & \\
\hline 11 & 1.138 & 1.005 & +0.133 & & & & & & \\
\hline 18 & 1.169 & 1.266 & -0.097 & & & & & & \\
\hline 19 & 1.421 & 1.319 & +0.102 & 1.403 & 1.310 & +0.093 & 1.4840 & 1.4840 & +0.049 \\
\hline 20 & 2.035 & 2.080 & -0.045 & & & & & & \\
\hline 22 & 4.319 & 4.540 & -0.221 & & & & & & \\
\hline
\end{tabular}


Table S2. ${ }^{13} \mathrm{C}$ NMR Data $(100 \mathrm{MHz})$ of $\mathbf{1 8}-23$ in $\mathrm{C}_{5} \mathrm{D}_{5} \mathrm{~N}, \mathrm{CDCl}_{3}$, or $\mathrm{CD}_{3} \mathrm{OD}$

\begin{tabular}{|c|c|c|c|c|c|c|}
\hline Pos & $18^{\mathrm{b}}$ & $19^{\mathrm{c}}$ & $20^{b}$ & $21^{b}$ & $22^{a}$ & $23^{b}$ \\
\hline 1 & $212.1(\mathrm{~s})$ & $213.2(\mathrm{~s})$ & $210.5(\mathrm{~s})$ & $210.6(\mathrm{~s})$ & $209.4(\mathrm{~s})$ & 209.9 (s) \\
\hline 2 & $32.2(\mathrm{t})$ & $43.6(t)$ & $39.1(\mathrm{t})$ & $39.6(t)$ & $41.1(\mathrm{t})$ & $40.1(\mathrm{t})$ \\
\hline 3 & $26.8(\mathrm{t})$ & 69.5 (d) & 77.5 (d) & 75.7 (d) & 78.8 (d) & 75.4 (d) \\
\hline 4 & 72.4 (d) & 78.6 (d) & 74.8 (d) & 75.4 (d) & 74.7 (d) & 75.8 (d) \\
\hline 5 & $66.6(\mathrm{~s})$ & $65.8(\mathrm{~s})$ & $65.4(\mathrm{~s})$ & $65.4(\mathrm{~s})$ & $65.1(\mathrm{~s})$ & $64.8(\mathrm{~s})$ \\
\hline 6 & $60.2(\mathrm{~d})$ & $60.4(\mathrm{~d})$ & $61.7(\mathrm{~d})$ & $61.6(\mathrm{~d})$ & $57.9(\mathrm{~d})$ & 60.0 (d) \\
\hline 7 & $30.5(\mathrm{t})$ & $31.4(\mathrm{t})$ & $30.3(\mathrm{t})$ & $30.4(\mathrm{t})$ & $31.5(\mathrm{t})$ & $30.9(\mathrm{t})$ \\
\hline 8 & 30.1 (d) & $32.0(\mathrm{~d})$ & $30.1(\mathrm{~d})$ & 30.1 (d) & $28.6(\mathrm{~d})$ & 27.9 (d) \\
\hline 9 & $42.1(\mathrm{~d})$ & $43.6(\mathrm{~d})$ & 41.9 (d) & $42.1(\mathrm{~d})$ & $43.6(\mathrm{~d})$ & 43.2 (d) \\
\hline 10 & $50.6(\mathrm{~s})$ & $51.9(\mathrm{~s})$ & $50.5(\mathrm{~s})$ & $50.4(\mathrm{~s})$ & $51.0(\mathrm{~s})$ & $50.5(\mathrm{~s})$ \\
\hline 11 & $24.2(\mathrm{t})$ & $24.4(\mathrm{t})$ & $24.1(\mathrm{t})$ & $24.2(\mathrm{t})$ & $21.4(\mathrm{t})$ & $21.4(\mathrm{t})$ \\
\hline 12 & $23.7(t)$ & $25.6(t)$ & $24.3(\mathrm{t})$ & $24.0(\mathrm{t})$ & $32.9(\mathrm{t})$ & $32.7(\mathrm{t})$ \\
\hline 13 & $138.6(\mathrm{~s})$ & $141.0(\mathrm{~s})$ & $138.5(\mathrm{~s})$ & $138.5(\mathrm{~s})$ & $42.4(\mathrm{~s})$ & $42.2(\mathrm{~s})$ \\
\hline 14 & $135.0(\mathrm{~s})$ & $136.1(\mathrm{~s})$ & $135.1(\mathrm{~s})$ & $135.1(\mathrm{~s})$ & 45.4 (d) & 45.1 (d) \\
\hline 15 & $40.8(\mathrm{t})$ & $41.6(t)$ & $40.5(\mathrm{t})$ & $40.9(\mathrm{t})$ & $27.7(t)$ & $27.5(t)$ \\
\hline 16 & 82.4 (d) & 82.8 (d) & 82.4 (d) & 82.3 (d) & $61.2(\mathrm{~d})$ & 61.4 (d) \\
\hline 17 & $53.3(\mathrm{~s})$ & $53.8(\mathrm{~s})$ & $53.3(\mathrm{~s})$ & $53.3(\mathrm{~s})$ & $71.0(\mathrm{~s})$ & $71.0(\mathrm{~s})$ \\
\hline 18 & $25.4(q)$ & $25.6(q)$ & $25.7(q)$ & $25.7(q)$ & $15.7(q)$ & $15.8(q)$ \\
\hline 19 & $15.2(q)$ & $14.9(q)$ & $15.5(\mathrm{q})$ & $15.5(q)$ & $15.3(\mathrm{q})$ & $15.5(q)$ \\
\hline 20 & 40.4 (d) & 42.7 (d) & $40.6(\mathrm{~d})$ & $40.6(\mathrm{~d})$ & $34.3(\mathrm{~d})$ & 33.9 (d) \\
\hline 21 & $12.6(q)$ & $12.1(q)$ & $12.5(\mathrm{q})$ & $12.5(q)$ & $12.6(q)$ & $12.3(q)$ \\
\hline 22 & 78.7 (d) & 80.4 (d) & 78.6 (d) & 78.7 (d) & $76.3(\mathrm{~d})$ & 75.8 (d) \\
\hline 23 & $34.5(\mathrm{t})$ & $33.6(t)$ & $34.3(\mathrm{t})$ & $34.3(\mathrm{t})$ & $33.6(t)$ & $33.5(\mathrm{t})$ \\
\hline 24 & $149.9(\mathrm{~s})$ & $153.2(\mathrm{~s})$ & $149.9(\mathrm{~s})$ & $149.8(\mathrm{~s})$ & $149.1(\mathrm{~s})$ & $148.4(\mathrm{~s})$ \\
\hline 25 & $121.7(\mathrm{~s})$ & $122.4(\mathrm{~s})$ & $121.7(\mathrm{~s})$ & $121.7(\mathrm{~s})$ & $121.9(\mathrm{~s})$ & $122.2(\mathrm{~s})$ \\
\hline 26 & $166.2(\mathrm{~s})$ & $169.8(\mathrm{~s})$ & $166.3(\mathrm{~s})$ & $166.2(\mathrm{~s})$ & $166.0(\mathrm{~s})$ & $166.1(\mathrm{~s})$ \\
\hline 27 & $12.4(q)$ & $12.5(\mathrm{q})$ & $12.3(q)$ & $12.3(\mathrm{q})$ & $12.7(\mathrm{q})$ & $12.4(q)$ \\
\hline 28 & $20.5(q)$ & $20.9(q)$ & $20.5(q)$ & $20.5(q)$ & $20.0(q)$ & $20.4(q)$ \\
\hline 1 , & & & $56.8(q)$ & $68.8(\mathrm{t})$ & $56.7(q)$ & $68.8(\mathrm{t})$ \\
\hline $2^{\prime}$ & & & & $31.8(\mathrm{t})$ & & $31.9(\mathrm{t})$ \\
\hline $3^{\prime}$ & & & & $19.2(\mathrm{t})$ & & $19.3(\mathrm{t})$ \\
\hline $4^{\prime}$ & & & & $13.8(q)$ & & $13.9(q)$ \\
\hline
\end{tabular}


Table S3. ${ }^{13} \mathrm{C}$ NMR Data (100 MHz) of $\mathbf{2 4}-\mathbf{2 7}$ in $\mathrm{C}_{5} \mathrm{D}_{5} \mathrm{~N}$

\begin{tabular}{ccccc}
\hline Pos & \multicolumn{1}{c}{$\mathbf{2 4}^{\mathrm{a}}$} & $\mathbf{2 5}^{\mathrm{a}}$ & $\mathbf{2 6}^{\mathrm{a}}$ & $\mathbf{2 7}^{\mathrm{a}}$ \\
\hline 1 & $210.0(\mathrm{~s})$ & $209.5(\mathrm{~s})$ & $209.6(\mathrm{~s})$ & $209.4(\mathrm{~s})$ \\
2 & $41.5(\mathrm{t})$ & $41.1(\mathrm{t})$ & $41.0(\mathrm{t})$ & $41.1(\mathrm{t})$ \\
3 & $79.3(\mathrm{~d})$ & $78.8(\mathrm{~d})$ & $78.8(\mathrm{~d})$ & $78.8(\mathrm{~d})$ \\
4 & $75.2(\mathrm{~d})$ & $74.7(\mathrm{~d})$ & $74.7(\mathrm{~d})$ & $74.7(\mathrm{~d})$ \\
5 & $65.5(\mathrm{~s})$ & $65.0(\mathrm{~s})$ & $65.0(\mathrm{~s})$ & $65.1(\mathrm{~s})$ \\
6 & $58.5(\mathrm{~d})$ & $58.0(\mathrm{~d})$ & $58.1(\mathrm{~d})$ & $57.9(\mathrm{~d})$ \\
7 & $32.5(\mathrm{t})$ & $31.6(\mathrm{t})$ & $32.0(\mathrm{t})$ & $31.4(\mathrm{t})$ \\
8 & $30.8(\mathrm{~d})$ & $28.5(\mathrm{~d})$ & $29.8(\mathrm{~d})$ & $28.6(\mathrm{~d})$ \\
9 & $43.5(\mathrm{~d})$ & $43.1(\mathrm{~d})$ & $43.1(\mathrm{~d})$ & $43.3(\mathrm{~d})$ \\
10 & $51.4(\mathrm{~s})$ & $50.9(\mathrm{~s})$ & $50.9(\mathrm{~s})$ & $51.0(\mathrm{~s})$ \\
11 & $22.0(\mathrm{t})$ & $22.5(\mathrm{t})$ & $21.4(\mathrm{t})$ & $21.5(\mathrm{t})$ \\
12 & $24.5(\mathrm{t})$ & $24.1(\mathrm{t})$ & $24.0(\mathrm{t})$ & $31.2(\mathrm{t})$ \\
13 & $48.6(\mathrm{~s})$ & $42.8(\mathrm{~s})$ & $49.0(\mathrm{~s})$ & $47.3(\mathrm{~s})$ \\
14 & $51.0(\mathrm{~d})$ & $54.9(\mathrm{~d})$ & $51.1(\mathrm{~d})$ & $57.6(\mathrm{~d})$ \\
15 & $32.5(\mathrm{t})$ & $21.4(\mathrm{t})$ & $32.5(\mathrm{t})$ & $35.8(\mathrm{t})$ \\
16 & $37.8(\mathrm{t})$ & $39.5(\mathrm{t})$ & $33.5(\mathrm{t})$ & $125.9(\mathrm{~d})$ \\
17 & $84.9(\mathrm{~s})$ & $56.6(\mathrm{~d})$ & $87.5(\mathrm{~s})$ & $158.4(\mathrm{~s})$ \\
18 & $15.0(\mathrm{q})$ & $13.7(\mathrm{q})$ & $15.4(\mathrm{q})$ & $17.5(\mathrm{q})$ \\
19 & $15.9(\mathrm{q})$ & $15.3(\mathrm{q})$ & $15.4(\mathrm{q})$ & $15.2(\mathrm{q})$ \\
20 & $44.1(\mathrm{~d})$ & $74.6(\mathrm{~d})$ & $77.7(\mathrm{~d})$ & $74.6(\mathrm{~d})$ \\
21 & $10.4(\mathrm{q})$ & $21.0(\mathrm{q})$ & $20.5(\mathrm{q})$ & $26.1(\mathrm{q})$ \\
22 & $79.7(\mathrm{~d})$ & $81.7(\mathrm{~d})$ & $81.2(\mathrm{~d})$ & $81.5(\mathrm{~d})$ \\
23 & $33.6(\mathrm{t})$ & $31.6(\mathrm{t})$ & $32.1(\mathrm{t})$ & $30.8(\mathrm{t})$ \\
24 & $151.0(\mathrm{~s})$ & $149.7(\mathrm{~s})$ & $150.5(\mathrm{~s})$ & $150.1(\mathrm{~s})$ \\
25 & $121.9(\mathrm{~s})$ & $121.6(\mathrm{~s})$ & $121.0(\mathrm{~s})$ & $121.4(\mathrm{~s})$ \\
26 & $167.3(\mathrm{~s})$ & $166.4(\mathrm{~s})$ & $166.2(\mathrm{~s})$ & $166.2(\mathrm{~s})$ \\
27 & $13.0(\mathrm{q})$ & $12.5(\mathrm{q})$ & $12.4(\mathrm{q})$ & $12.6(\mathrm{q})$ \\
\hline & $20.5(\mathrm{q})$ & $20.1(\mathrm{q})$ & $20.1(\mathrm{q})$ & $20.1(\mathrm{q})$ \\
\hline & $57.1(\mathrm{q})$ & $56.7(\mathrm{q})$ & $56.6(\mathrm{q})$ & $56.6(\mathrm{q})$
\end{tabular}

${ }^{\mathrm{a}}$ All assignments were confirmed by DEPT, COSY, and HMQC 
Table S4. ${ }^{1} \mathrm{H}$ NMR Data (400 MHz) of $\mathbf{1 8}-21$ in $\mathrm{CDCl}_{3}$, or $\mathrm{CD}_{3} \mathrm{OD}$

\begin{tabular}{|c|c|c|c|c|c|c|c|c|}
\hline Pos & 18 & & 19 & & 203 & & 21 & \\
\hline \multirow[t]{2}{*}{2} & 2.79 & $(\mathrm{ddd}, 15.2,8.0,7.6)$ & 2.58 & $(\mathrm{dd}, 12.4,2.8)$ & 2.63 & $(\mathrm{dd}, 14.8,4.0)$ & 2.62 & $(\mathrm{dd}, 14.4,4.0)$ \\
\hline & 2.50 & (m) & 2.89 & $(\mathrm{dd}, 12.4,6.0)$ & 3.14 & $(\mathrm{dd}, 14.8,4.8)$ & 3.14 & $(\mathrm{dd}, 14.4,5.0)$ \\
\hline 3 & 1.96 & $(\mathrm{~m})$ & 4.06 & $(\mathrm{ddd}, 6.0,2.8,2.8)$ & 3.75 & $(\mathrm{ddd}, 4.8,4.0,3.4)$ & 3.82 & (ddd, 5.0, 4.0, 3.6) \\
\hline 4 & 3.54 & $(\mathrm{dd}, 4.0,3.6)$ & 3.31 & $(\mathrm{~d}, 2.8)$ & 3.51 & $(\mathrm{~d}, 3.4)$ & 3.49 & $(\mathrm{~d}, 3.6)$ \\
\hline 6 & 3.24 & (brs) & 3.28 & (br s) & 3.30 & (br s) & 3.29 & (br s) \\
\hline \multirow[t]{2}{*}{7} & 1.25 & $(\mathrm{~m})$ & 1.41 & $(\mathrm{~d}, 10.8)$ & 1.25 & (m) & 1.23 & $(\mathrm{dd}, 14.4,1.2)$ \\
\hline & 2.35 & $(\mathrm{ddd}, 14.4,4.0,2.0)$ & 2.32 & $(\mathrm{ddd}, 10.8,2.4,1.2)$ & 2.32 & $(\mathrm{ddd}, 14.4,4.0,2.4)$ & 2.33 & $(\mathrm{ddd}, 14.4,3.2,2.4)$ \\
\hline 8 & 2.10 & (m) & 2.07 & (m) & 2.07 & (m) & 2.07 & (m) \\
\hline 9 & 1.40 & $(\mathrm{dd}, 12.4,11.2)$ & 1.44 & $(\mathrm{ddd}, 9.2,8.0,1.2)$ & 1.44 & $(\mathrm{ddd}, 10.8,10.0,2.0)$ & 1.42 & $(\mathrm{ddd}, 10.8,10.0,1.6)$ \\
\hline \multirow[t]{2}{*}{11} & 1.06 & $(\mathrm{~m})$ & 1.05 & $(\mathrm{dd}, 10.0,4.0)$ & 1.05 & $(\mathrm{dd}, 12.0,4.0)$ & 1.05 & $(\mathrm{dd}, 12.0,4.4)$ \\
\hline & 2.04 & (m) & 1.68 & $(\mathrm{dd}, 10.0,1.2)$ & 2.02 & (m) & 2.02 & (m) \\
\hline \multirow[t]{2}{*}{12} & 1.77 & $(\mathrm{~m})$ & 1.85 & (m) & 1.83 & (m) & 1.82 & (m) \\
\hline & 1.77 & (m) & 2.07 & (m) & 1.83 & (m) & 1.82 & (m) \\
\hline \multirow[t]{2}{*}{15} & 2.43 & (m) & 2.20 & (m) & 2.42 & (m) & 2.42 & (m) \\
\hline & 2.43 & $(\mathrm{~m})$ & 2.38 & $(\mathrm{dd}, 12.8,7.2)$ & 2.42 & (m) & 2.42 & (m) \\
\hline 16 & 4.12 & $(\mathrm{t}, 7.6)$ & 4.10 & $(\mathrm{t}, 6.8)$ & 4.13 & $(\mathrm{dd}, 8.4,7.6)$ & 4.13 & $(\mathrm{dd}, 8.0,8.0,8.0)$ \\
\hline 18 & 1.12 & (s) & 1.09 & (s) & 1.12 & (s) & 1.12 & (s) \\
\hline 19 & 1.30 & (s) & 1.20 & (s) & 1.30 & (s) & 1.29 & (s) \\
\hline 20 & 2.16 & $(\mathrm{qd}, 7.2,3.2)$ & 2.20 & (m) & 2.17 & $(\mathrm{qd}, 7.2,6.6)$ & 2.17 & $(\mathrm{qd}, 7.2,6.6)$ \\
\hline 21 & 0.98 & $(\mathrm{~d}, 7.2)$ & 1.02 & $(\mathrm{~d}, 6.0)$ & 0.98 & $(d, 7.2)$ & 0.98 & $(\mathrm{~d}, 7.2)$ \\
\hline 22 & 4.35 & $(\mathrm{ddd}, 12.4,7.2,3.2)$ & 4.70 & $(\mathrm{ddd}, 10.4,3.2,2.4)$ & 4.37 & $(\mathrm{ddd}, 12.8,6.6,3.2)$ & 4.37 & $(\mathrm{ddd}, 12.8,6.6,3.2)$ \\
\hline \multirow[t]{2}{*}{23} & 2.48 & (m) & 2.54 & $(\mathrm{dd}, 12.4,10.4)$ & 2.48 & $(\mathrm{dd}, 17.6,12.8)$ & 2.44 & $(\mathrm{dd}, 17.2,12.8)$ \\
\hline & 2.24 & (m) & 2.24 & $(\mathrm{dd}, 12.4,2.4)$ & 2.22 & $(\mathrm{dd}, 17.6,3.2)$ & 2.20 & $(\mathrm{dd}, 17.2,2.4)$ \\
\hline 27 & 1.87 & (s) & 1.82 & (s) & 1.86 & (s) & 1.85 & (s) \\
\hline 28 & 1.94 & (s) & 1.96 & (s) & 1.94 & (s) & 1.93 & (s) \\
\hline \multirow[t]{2}{*}{1 , } & & & & & 3.34 & (s) & 3.39 & $(\mathrm{td}, 9.2,6.4)$ \\
\hline & & & & & & & 3.50 & $(\mathrm{td}, 9.2,6.4)$ \\
\hline 2 ' & & & & & & & 1.48 & (m) \\
\hline $3^{\prime}$ & & & & & & & 1.30 & (m) \\
\hline 4, & & & & & & & 0.88 & $(\mathrm{t}, 8.8)$ \\
\hline
\end{tabular}

${ }^{\mathrm{a}}=$ measured in $\mathrm{CDCl}_{3} ;{ }^{\mathrm{b}}=$ measured in $\mathrm{CD}_{3} \mathrm{OD}$; all assignments were confirmed by DEPT, COSY, and HMQC. 
Table S5. ${ }^{1} \mathrm{H}$ NMR Data (400 MHz) of 22 and 23 in $\mathrm{C}_{5} \mathrm{D}_{5} \mathrm{~N}$ or $\mathrm{CDCl}_{3}$

\begin{tabular}{|c|c|c|}
\hline Pos & $22^{\mathrm{a}}$ & $23^{b}$ \\
\hline \multirow[t]{2}{*}{2} & $2.97(\mathrm{dd}, 15.2,3.2)$ & $2.60(\mathrm{dd}, 15.2,3.2)$ \\
\hline & $3.15(\mathrm{dd}, 15.2,8.0)$ & $2.94(\mathrm{dd}, 15.2,6.4)$ \\
\hline 3 & $3.93(\mathrm{~m})$ & $3.77(\mathrm{ddd}, 6.4,3.2,2.8)$ \\
\hline 4 & $3.89(\mathrm{~m})$ & $3.49(\mathrm{~d}, 2.8)$ \\
\hline 6 & $3.36(\mathrm{br} \mathrm{s})$ & 3.21 (br s) \\
\hline \multirow[t]{2}{*}{7} & $1.32(\mathrm{~m})$ & $1.35(\mathrm{~m})$ \\
\hline & $2.11(\mathrm{~m})$ & $2.18(\mathrm{~m})$ \\
\hline 8 & $1.61(\mathrm{~m})$ & $1.44(\mathrm{~m})$ \\
\hline 9 & $1.32(\mathrm{~m})$ & $1.19(\mathrm{td}, 12.4,8.0)$ \\
\hline \multirow[t]{2}{*}{11} & $1.53(\mathrm{~m})$ & $1.46(\mathrm{~m})$ \\
\hline & $1.53(\mathrm{~m})$ & $1.46(\mathrm{~m})$ \\
\hline \multirow[t]{2}{*}{12} & $1.39(\mathrm{~m})$ & $1.43(\mathrm{~m})$ \\
\hline & $1.59(\mathrm{~m})$ & $1.60(\mathrm{~m})$ \\
\hline 14 & $1.28(\mathrm{~m})$ & $1.16(\mathrm{~m})$ \\
\hline \multirow[t]{2}{*}{15} & $1.14(\mathrm{dd}, 12.8,12.0)$ & $1.26(\mathrm{~m})$ \\
\hline & $1.80(\mathrm{dd}, 12.8,6.0)$ & $1.86(\mathrm{~m})$ \\
\hline 16 & $3.60(\mathrm{br} \mathrm{s})$ & 3.46 (br s) \\
\hline 18 & $0.87(\mathrm{~s})$ & $0.84(\mathrm{~s})$ \\
\hline 19 & $1.69(\mathrm{~s})$ & $1.29(\mathrm{~s})$ \\
\hline 20 & $2.45(\mathrm{dq}, 8.0,6.8)$ & $2.42(\mathrm{dq}, 8.8,7.2)$ \\
\hline 21 & $1.02(\mathrm{~d}, 6.8)$ & $0.99(\mathrm{~d}, 7.2)$ \\
\hline 22 & $3.93(\mathrm{~m})$ & $3.86(\mathrm{ddd}, 12.0,8.8,3.6)$ \\
\hline \multirow[t]{2}{*}{23} & $2.22(\mathrm{dd}, 17.2,12.0)$ & $2.30(\mathrm{dd}, 19.2,12.0)$ \\
\hline & $2.09(\mathrm{dd}, 17.2,3.2)$ & $2.15(\mathrm{~m})$ \\
\hline 27 & $1.86(\mathrm{~s})$ & $1.86(\mathrm{~s})$ \\
\hline 28 & $1.92(\mathrm{~s})$ & $1.92(\mathrm{~s})$ \\
\hline \multirow[t]{2}{*}{1 ' } & $3.33(\mathrm{~s})$ & $3.39(\mathrm{dt}, 8.8,6.4)$ \\
\hline & & $3.48(\mathrm{dt}, 8.8,6.4)$ \\
\hline 2 ' & & $1.48(\mathrm{~m})$ \\
\hline $3^{\prime}$ & & $1.34(\mathrm{~m})$ \\
\hline $4^{\prime}$ & & $0.91(\mathrm{t}, 7.2)$ \\
\hline
\end{tabular}

$\overline{\mathrm{a}}=$ measured in $\mathrm{C}_{5} \mathrm{D}_{5} \mathrm{~N},{ }^{b}=$ measured in $\mathrm{CDCl}_{3}$; all assignments were confirmed by DEPT, COSY, and HMQC; OH signals 23: $\delta_{\mathrm{H}}=7.90$ (br d, 2.4, OH-4) 
Table S6. ${ }^{1} \mathrm{H}$ NMR Data $(400 \mathrm{MHz})$ of $24-27$ in $\mathrm{C}_{5} \mathrm{D}_{5} \mathrm{~N}$

\begin{tabular}{|c|c|c|c|c|}
\hline Pos & $24^{\mathrm{a}}$ & $25^{\mathrm{a}}$ & $26^{\mathrm{a}}$ & $27^{\mathrm{a}}$ \\
\hline \multirow[t]{2}{*}{2} & $2.87(\mathrm{dd}, 15.4,3.5)$ & $2.97(\mathrm{dd}, 15.2,3.2)$ & $2.91(\mathrm{dd}, 15.2,3.2)$ & $2.97(\mathrm{dd}, 15.6,4.0)$ \\
\hline & $3.08(\mathrm{dd}, 15.4,7.5)$ & $3.15(\mathrm{dd}, 15.2,8.0)$ & $3.09(\mathrm{dd}, 15.2,8.0)$ & $3.11(\mathrm{dd}, 15.6,8.0)$ \\
\hline 3 & $3.92(\mathrm{dd}, 7.5,3.5)$ & 3.95 (ddd, $8.0,5.8,3.2)$ & $3.91(\mathrm{ddd}, 8.0,3.2,3.2)$ & $3.93(\mathrm{ddd}, 8.0,4.0,3.2)$ \\
\hline 4 & $3.90($ br s) & $3.92(\mathrm{dd}, 5.8,2.4)$ & $3.90(\mathrm{~d}, 3.2)$ & $3.90(\mathrm{~d}, 3.2)$ \\
\hline 6 & 3.37 (br s) & 3.41 (br s) & 3.37 (br s) & $3.40(\mathrm{br} \mathrm{s})$ \\
\hline \multirow[t]{2}{*}{7} & $1.37(\mathrm{~m})$ & $2.27(\mathrm{~m})$ & $1.39(\mathrm{~m})$ & $1.39(\mathrm{~m})$ \\
\hline & $2.21(\mathrm{dd}, 4.5,2.5)$ & $2.54(\mathrm{~m})$ & $2.19(\mathrm{~m})$ & $2.18(\mathrm{~m})$ \\
\hline 8 & $1.61(\mathrm{~m})$ & $1.56(\mathrm{~m})$ & $1.65(\mathrm{~m})$ & $1.60(\mathrm{~m})$ \\
\hline 9 & $1.34(\mathrm{~m})$ & $1.28(\mathrm{~m})$ & $1.44(\mathrm{~m})$ & $1.42(\mathrm{~m})$ \\
\hline \multirow[t]{2}{*}{11} & $1.57(\mathrm{~m})$ & $1.75(\mathrm{~m})$ & $1.64(\mathrm{~m})$ & $1.75(\mathrm{~m})$ \\
\hline & $1.61(\mathrm{~m})$ & $2.18(\mathrm{~m})$ & $1.69(\mathrm{~m})$ & $2.18(\mathrm{~m})$ \\
\hline \multirow[t]{2}{*}{12} & $1.08(\mathrm{~m})$ & $1.11(\mathrm{~m})$ & $1.17(\mathrm{~m})$ & $1.63(\mathrm{~m})$ \\
\hline & $1.72(\mathrm{~m})$ & $1.61(\mathrm{~m})$ & $1.70(\mathrm{~m})$ & $2.20(\mathrm{~m})$ \\
\hline 14 & $1.92(\mathrm{~m})$ & $1.85(\mathrm{~m})$ & $2.03(\mathrm{dq}, 7.6,3.6)$ & $1.50(\mathrm{~m})$ \\
\hline \multirow[t]{2}{*}{15} & $1.73(\mathrm{~m})$ & $1.50(\mathrm{~m})$ & $1.90(\mathrm{~m})$ & $1.85(\mathrm{~m})$ \\
\hline & $1.97(\mathrm{~m})$ & $1.54(\mathrm{~m})$ & $2.16(\mathrm{~m})$ & $2.05(\mathrm{~m})$ \\
\hline \multirow[t]{2}{*}{16} & $1.89(\mathrm{~m})$ & $1.23(\mathrm{~m})$ & $2.16(\mathrm{~m})$ & $6.04(\mathrm{~d}, 2.0)$ \\
\hline & $1.98(\mathrm{~m})$ & $1.96(\mathrm{~m})$ & $2.99(\mathrm{ddd}, 12.0,3.2,2.8)$ & \\
\hline 18 & $0.73(\mathrm{~s})$ & $0.82(\mathrm{~m})$ & & \\
\hline 19 & $1.76(\mathrm{~s})$ & $0.97(\mathrm{~s})$ & $1.20(\mathrm{~s})$ & $1.03(\mathrm{~s})$ \\
\hline 20 & $2.34(\mathrm{qd}, 7.0,3.2)$ & $1.71(\mathrm{~s})$ & $1.76(\mathrm{~s})$ & $1.73(\mathrm{~s})$ \\
\hline 21 & $1.20(\mathrm{~d}, 7.0)$ & $1.39(\mathrm{~s})$ & $1.53(\mathrm{~s})$ & $1.55(\mathrm{~s})$ \\
\hline 22 & $4.77(\mathrm{ddd}, 13.0,3.0,2.4)$ & $4.38(\mathrm{dd}, 12.8,3.6)$ & $5.01(\mathrm{dd}, 14.8,3.2)$ & $4.55(\mathrm{dd}, 12.8,3.6)$ \\
\hline \multirow[t]{2}{*}{23} & $2.44(t, 14.5)$ & $1.40(\mathrm{~m})$ & $2.43(\mathrm{dd}, 18.0,3.2)$ & $2.34(\mathrm{dd}, 18.0,3.6)$ \\
\hline & $2.65(\mathrm{dd}, 18.4,3.6)$ & $2.19(\mathrm{~m})$ & $2.73(\mathrm{dd}, 18.0,14.8)$ & $2.82(\mathrm{dd}, 18,12.8)$ \\
\hline 27 & $1.94(\mathrm{~s})$ & $1.93(\mathrm{~s})$ & $1.85(\mathrm{~s})$ & $1.91(\mathrm{~s})$ \\
\hline 28 & $1.65(\mathrm{~s})$ & $1.81(\mathrm{~s})$ & $1.67(\mathrm{~s})$ & $1.74(\mathrm{~s})$ \\
\hline 1 ' & $3.29(\mathrm{~s})$ & $3.37(\mathrm{~s})$ & $3.30(\mathrm{~s})$ & $3.35(\mathrm{~s})$ \\
\hline
\end{tabular}

${ }^{a}$ all assignments were confirmed by DEPT, COSY, and HMQC; OH signals 24: $\delta_{\mathrm{H}}=7.91$ (br s); 25: $\delta_{\mathrm{H}}=7.92\left(\right.$ br s, OH-4), $6.12\left(\right.$ br s, OH-20); 26: $\delta_{\mathrm{H}}=7.91$ (br s, OH-4), 5.90 (br s, $\mathrm{OH}-20$ ), 5.31 (br s, OH-17); 27: $\delta_{\mathrm{H}}=7.93$ (br s, OH-4), 6.37 (br s, OH-20). 

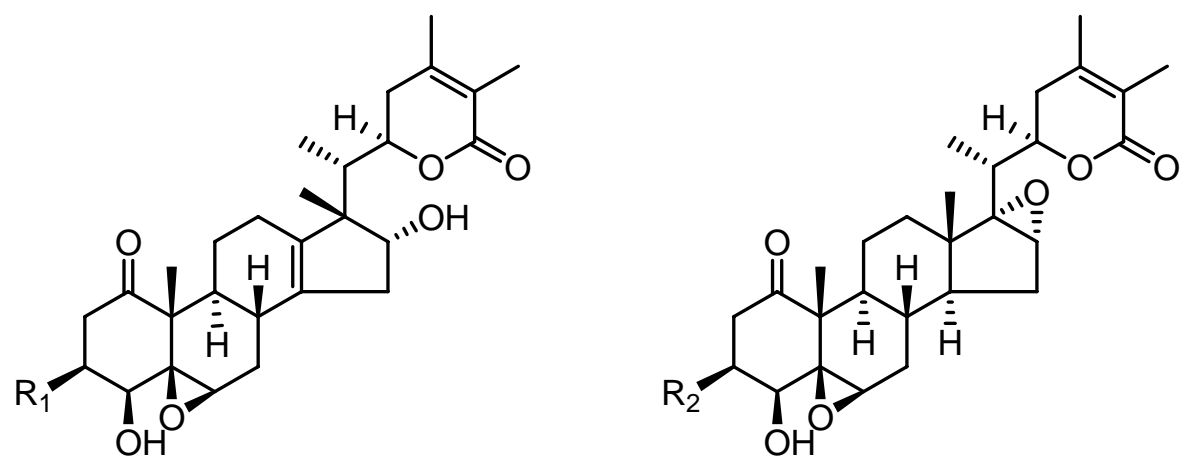

18: $R_{1}=H$

19: $R_{1}=O H$

22: $\mathbf{R}_{2}=\mathrm{OCH}_{3}$

20: $\mathrm{R}_{1}=\mathrm{OCH}_{3}$

23: $\mathbf{R}_{2}=O \mathrm{OC}_{4} \mathrm{H}_{9}$

21: $\mathrm{R}_{1}=\mathrm{OC}_{4} \mathrm{H}_{9}$

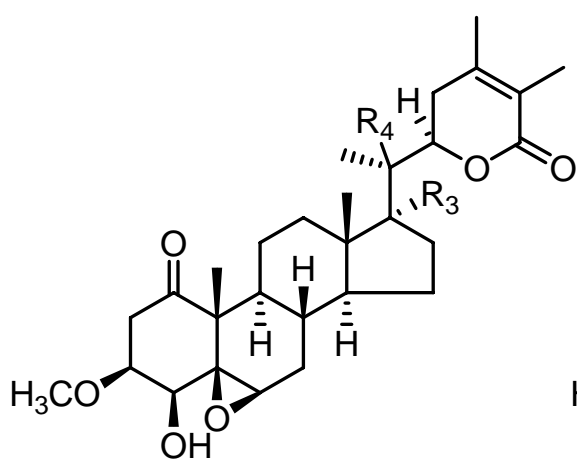

24: $\mathrm{R}_{3}=\mathrm{OH}, \mathrm{R}_{4}=\mathrm{H}$

25: $\mathrm{R}_{3}=\mathrm{H}, \mathrm{R}_{4}=\mathrm{OH}$

26: $\mathrm{R}_{3}=\mathrm{OH}, \mathrm{R}_{4}=\mathrm{OH}$

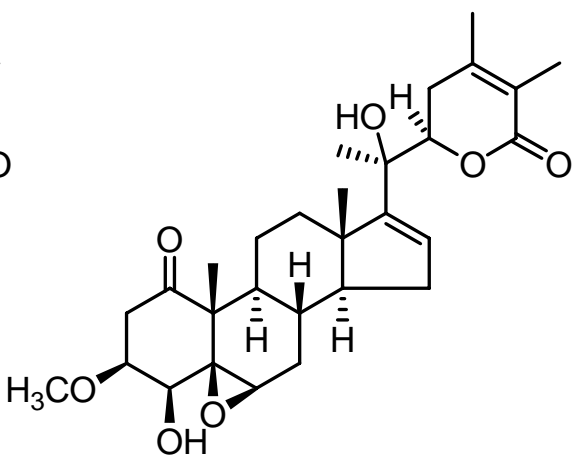

27

Figure S1. The Structures of Artificial Components (18-27) from Tubocapsicum anomalum 


\section{Additional Experimental Details.}

Extraction and Isolation. The air-dried stems and leaves $(2.5 \mathrm{~kg}$, Part A) and roots $(1.2 \mathrm{~kg}$, Part B) of T. anomalum were extracted separately with $\mathrm{MeOH}$ at $\mathrm{rt}$. The $\mathrm{MeOH}$ extract of Part A was partitioned between EtOAc/ $\mathrm{H}_{2} \mathrm{O}$ to yield EtOAc and $\mathrm{H}_{2} \mathrm{O}$ extracts. The $\mathrm{H}_{2} \mathrm{O}$ extracts were further partitioned with $n-\mathrm{BuOH}$ to give $n$ - $\mathrm{BuOH}$ and $\mathrm{H}_{2} \mathrm{O}$ extracts. These extracts were evaporated to give dark-green viscous residues. The residue from the EtOAc extract was further separated on a Si gel column $(230 \sim 400$ mesh, $5 \times 20 \mathrm{~cm})$ eluting with a gradient of $n$-hexane $/ \mathrm{CHCl}_{3} / \mathrm{MeOH}$ to give 16 fractions (A1-A16). The mother liquor of fractions A8, A11 and A12 were combined with fractions A9 and A10 to yield fraction TAEW, and then partitioned with $\mathrm{H}_{2} \mathrm{O} / \mathrm{MeOH} / \mathrm{CHCl}_{3}=1: 4: 5$ to yield fractions TAEWM and TAEWC. Fraction TAEWM was separated by preparative reverse-phase HPLC $\left(\mathrm{MeOH} / \mathrm{H}_{2} \mathrm{O}=65: 35\right.$, flow rate: $3.0 \mathrm{~mL} / \mathrm{min}$ ) to obtain six subfractions (TAEWM-1 6). Subfraction TAEWM-3 was separated by preparative reverse-phase $\mathrm{HPLC}\left(250 \times 10 \mathrm{~mm}, \mathrm{MeOH} / \mathrm{H}_{2} \mathrm{O}=45: 55\right.$, flow rate: $\left.2.0 \mathrm{~mL} / \mathrm{min}\right)$ and gave tubocapsenolide B (18, $2.3 \mathrm{mg}$, R.T. = $62.5 \mathrm{~min})$. Subfraction TAEWM-5 was purified by preparative reverse-phase $\operatorname{HPLC}\left(250 \times 4.6 \mathrm{~mm}, \mathrm{MeOH} / \mathrm{H}_{2} \mathrm{O}=50: 50\right.$, flow rate: $1.0 \mathrm{~mL} / \mathrm{min})$ and gave tubocapsenolide D $(\mathbf{2 0}, 20.0 \mathrm{mg}, \mathrm{R} . \mathrm{T} .=24.0 \mathrm{~min})$.

The $n$ - $\mathrm{BuOH}$ extract was further partitioned between $\mathrm{CHCl}_{3}$ and $\mathrm{H}_{2} \mathrm{O}$, and then evaporated to give a dark-green viscous residue. The $\mathrm{CHCl}_{3}$ extract was further separated on a Sephadex LH-20 column eluting with $\mathrm{MeOH}$ to give seven fractions (B1 - B7). Fraction B4 (276.5 mg) was further separated on a Si gel column $(230 \sim 400$ mesh, $2.5 \times 27 \mathrm{~cm})$ eluting with $\mathrm{CHCl}_{3}$ to yield 21 subfractions (B4A - B4U). Subfraction B4E $(17.15 \mathrm{mg})$ was purified by preparative reverse-phase $\mathrm{HPLC}\left(250 \times 10 \mathrm{~mm}, \mathrm{MeOH} / \mathrm{H}_{2} \mathrm{O}=75: 25\right.$, flow rate: $\left.2.0 \mathrm{~mL} / \mathrm{min}\right)$ and gave tubocapsanolide C $(23,2.7 \mathrm{mg}, \mathrm{R} . \mathrm{T} .=42.5 \mathrm{~min})$. Subfractions B4J $(6.22 \mathrm{mg})$ and B4Q (11.1 mg) were purified by preparative reverse-phase $\operatorname{HPLC}\left(250 \times 4.6 \mathrm{~mm}, \mathrm{MeOH} / \mathrm{H}_{2} \mathrm{O}=70: 30\right.$, flow rate: $1.0 \mathrm{~mL} / \mathrm{min})$ to yield tubocapsenolide $\mathrm{E}(\mathbf{2 1}, 2.7 \mathrm{mg}, \mathrm{R} . \mathrm{T} .=14.3 \mathrm{~min})$. Fraction B5 (278.3 mg) was further separated on a Si gel column $(230 \sim 400$ mesh, $2.5 \times 27 \mathrm{~cm})$ eluting with $\mathrm{CHCl}_{3} / \mathrm{MeOH}=150: 1$ to yield 22 subfractions (B54A - B5V). Subfraction B5I (15.7 mg) was purified by preparative reverse-phase $\operatorname{HPLC}\left(250 \times 10 \mathrm{~mm}, \mathrm{MeOH} / \mathrm{H}_{2} \mathrm{O}=60: 40\right.$, flow rate: 2.0 $\mathrm{mL} / \mathrm{min})$ and gave tubocapsanolide B $(22,8.3 \mathrm{mg}$, R.T. $=40.8 \mathrm{~min})$. Subfractions B5K (19.66 $\mathrm{mg})$ and B5R $(11.1 \mathrm{mg})$ were purified by preparative reverse-phase HPLC $(250 \times 10 \mathrm{~mm}$, 
$\mathrm{MeOH} / \mathrm{H}_{2} \mathrm{O}=65: 35$, flow rate: $\left.2.0 \mathrm{~mL} / \mathrm{min}\right)$ to yield tubocapsenolide $\mathrm{C}(\mathbf{1 9}, 6.72 \mathrm{mg}$, R.T. $=$ $17.6 \mathrm{~min})$.

The $\mathrm{MeOH}$ extract of Part B was partitioned between EtOAc/ $\mathrm{H}_{2} \mathrm{O}$ to yield EtOAc and $\mathrm{H}_{2} \mathrm{O}$ extracts. The $\mathrm{H}_{2} \mathrm{O}$ extracts were further partitioned with $n$ - $\mathrm{BuOH}$ to give $n-\mathrm{BuOH}$ and $\mathrm{H}_{2} \mathrm{O}$ extracts. Additionally, the EtOAc extracts were further partitioned with $n$-hexane and $\mathrm{MeOH}$ to give $n$-hexane and $\mathrm{MeOH}$ extracts. The $\mathrm{MeOH}$ extracts were separated on a Sephadex LH-20 column $(1.6 \times 28 \mathrm{~cm})$ eluting with $\mathrm{MeOH}$ to give five fractions $(\mathrm{C} 1-\mathrm{C} 5)$. The $\mathrm{C} 3$ fraction $(807 \mathrm{mg}$ ) was further separated by Si gel column $(230 \sim 400$ mesh, $5 \times 20 \mathrm{~cm})$ gradient eluting with $\mathrm{CHCl}_{3} / \mathrm{MeOH}$ to give 28 fractions (C3-1 28). Fraction $\mathrm{C} 3-3$ was separated by preparative reverse-phase $\mathrm{HPLC}\left(250 \times 10 \mathrm{~mm}, \mathrm{MeOH} / \mathrm{H}_{2} \mathrm{O}=65: 35\right.$, flow rate: $\left.2.0 \mathrm{~mL} / \mathrm{min}\right)$ to give iso-tubocapsanolide $\mathrm{G}(\mathbf{2 5}, 2.20 \mathrm{mg}$, R.T. $=34.0 \mathrm{~min})$, and six subfractions $(\mathrm{C} 3-3 \mathrm{~A} \sim \mathrm{H})$. Subfraction C3-3C was separated by recycle reverse-phase HPLC $(250 \times 10 \mathrm{~mm}$, $\mathrm{MeOH} / \mathrm{H}_{2} \mathrm{O}=65: 35$, flow rate: $2.0 \mathrm{~mL} / \mathrm{min}$, five times) to yield 20-hydroxy-tubocapsanolide $\mathrm{G}$ (SI9, $2.60 \mathrm{mg}$, R.T. $=18.1 \mathrm{~min}$ ). Subfraction C3-3D was separated by recycle reverse-phase HPLC $\left(250 \times 10 \mathrm{~mm}, \mathrm{MeOH} / \mathrm{H}_{2} \mathrm{O}=65: 35\right.$, flow rate: $2.0 \mathrm{~mL} / \mathrm{min}$, four times $)$ to afford tubocapsanolide H $(27,2.90 \mathrm{mg}$, R.T. $=24.2 \mathrm{~min})$ and tubocapsanolide $\mathrm{G}(\mathbf{2 4}, 1.00 \mathrm{mg}$, R.T. $=$ $25.8 \mathrm{~min})$.

Tubocapsenolide B (18): white powder; mp. $133-135^{\circ} \mathrm{C} ;[\alpha]_{\mathrm{D}}^{26.4}-120^{\circ}(c 0.1, \mathrm{MeOH})$;

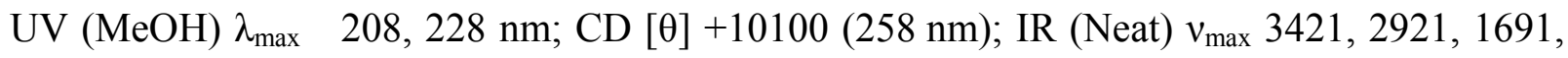
$1380,1130 \mathrm{~cm}^{-1} ;{ }^{1} \mathrm{H}$ and ${ }^{13} \mathrm{C}$ NMR data, see Table S2 and S4; HRESI-MS $\mathrm{m} / \mathrm{z} 493.2568$ $[\mathrm{M}+\mathrm{Na}]^{+}$(calcd. 493.2561).

Tubocapsenolide C (19): white powder; mp. $226-228{ }^{\circ} \mathrm{C} ;[\alpha]_{\mathrm{D}}^{24.4}-12.6$ (c 0.1, MeOH);

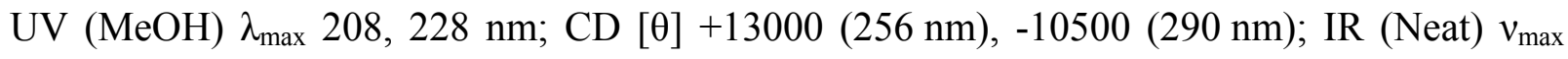
$3411,2919,1681,1135,1060 \mathrm{~cm}^{-1} ;{ }^{1} \mathrm{H}$ and ${ }^{13} \mathrm{C}$ NMR data, see Table S2 and S4; HRESI-MS $\mathrm{m} / \mathrm{z} 509.2516[\mathrm{M}+\mathrm{Na}]^{+}$(calcd. 509.2510).

Tubocapsenolide D (20): white powder; mp. $124-126{ }^{\circ} \mathrm{C}$; $[\alpha]_{\mathrm{D}}^{24.3}-46.1$ (c $\left.0.1, \mathrm{MeOH}\right)$;

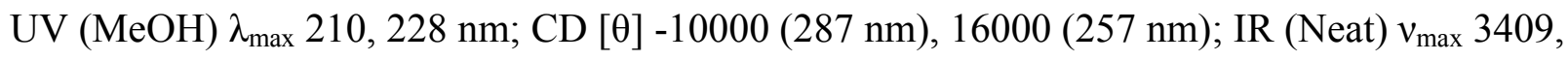


2921, 1691, 1384, 1135, $1097 \mathrm{~cm}^{-1} ;{ }^{1} \mathrm{H}$ and ${ }^{13} \mathrm{C}$ NMR data, see Table S2 and S4; HRESI-MS $m / z 523.2672[\mathrm{M}+\mathrm{Na}]^{+}($calcd. 523.2666).

Tubocapsenolide E (21): white powder; mp. $104-106{ }^{\circ} \mathrm{C}$; $[\alpha]_{\mathrm{D}}^{24.3}-49.1$ (c 0.1, MeOH); UV (MeOH) $\lambda_{\max } 210,228 \mathrm{~nm}$; CD [0] +12100 (256 nm), -11000 (290 nm); IR (Neat) $v_{\max }$ 3413, 2917, 1702, 1687, 1394, 1132, $1091 \mathrm{~cm}^{-1} ;{ }^{1} \mathrm{H}$ and ${ }^{13} \mathrm{C}$ NMR data, see Table S2 and S4; HRESI-MS $m / z 565.3193[\mathrm{M}+\mathrm{Na}]^{+}$(calcd. 565.3136).

Tubocapsanolide B (22): white powder; mp. $225-227{ }^{\circ} \mathrm{C} ;[\alpha]_{\mathrm{D}}^{24.4}-43.7$ (c 0.1, MeOH);

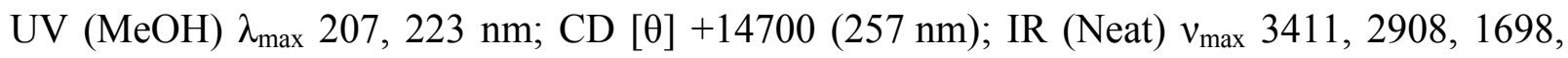
1382, $1126 \mathrm{~cm}^{-1} ;{ }^{1} \mathrm{H}$ and ${ }^{13} \mathrm{C}$ NMR data, see Table S2 and S5; HRESI-MS $m / z 523.2672$ $[\mathrm{M}+\mathrm{Na}]^{+}$(calcd. 523.2666).

Tubocapsanolide C (23): white powder; mp. $223-225{ }^{\circ} \mathrm{C} ;[\alpha]_{\mathrm{D}}^{24.4}-15.7$ (c 0.1, MeOH);

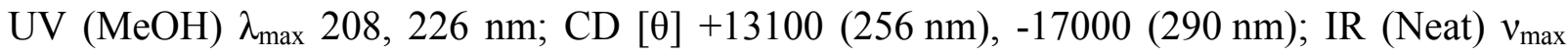
3388, 2931, 1702, 1687, 1380, $1095 \mathrm{~cm}^{-1} ;{ }^{1} \mathrm{H}$ and ${ }^{13} \mathrm{C}$ NMR data, see Table S2 and S5; HRESI-MS $m / z 565.3137[\mathrm{M}+\mathrm{Na}]^{+}$(calcd. 565.3136).

Tubocapsanolide G (24): white powder; mp. $218-220^{\circ} \mathrm{C}$; $[\alpha]_{\mathrm{D}}^{24.8}-46.1(c \quad 0.03, \mathrm{MeOH})$;

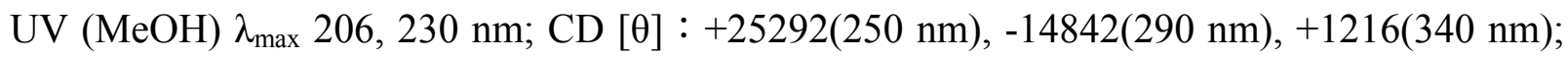
IR (Neat) $v_{\max } 3439,2922,1690,1129,1096,750 \mathrm{~cm}^{-1} ;{ }^{1} \mathrm{H}$ and ${ }^{13} \mathrm{C}$ NMR data, see Table S3 and S6; HRESI-MS $m / z 525.2830[\mathrm{M}+\mathrm{Na}]^{+}$(calcd. 525.2823).

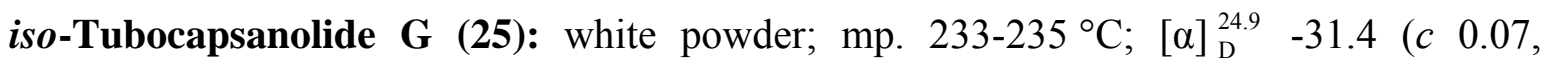

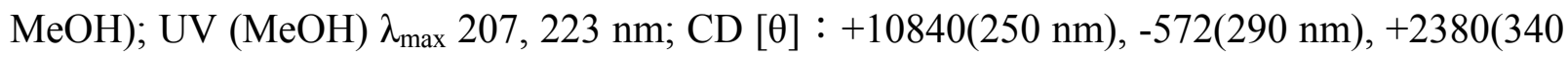
$\mathrm{nm}$ ); IR (Neat) $v_{\max } 3424,2929,1705,1384,1129,1096,753 \mathrm{~cm}^{-1} ;{ }^{1} \mathrm{H}$ and ${ }^{13} \mathrm{C}$ NMR data, see Table S3 and S6; HRESI-MS $m / z$ 525.2830 [M+Na] ${ }^{+}$(calcd. 525.2823).

20-Hydroxy-tubocapsanolide G (26): white powder; mp. 208-210 ${ }^{\circ} \mathrm{C}$; $[\alpha]_{\mathrm{D}}^{25.1}-42.0(c 0.1$, $\mathrm{MeOH}) ; \mathrm{UV}(\mathrm{MeOH}) \lambda_{\max } 206,228 \mathrm{~nm}$; CD [0] : +9590(250 nm), -2977(290 nm), +1243 (340 $\mathrm{nm}$ ); IR (Neat) $v_{\max } 3461,2922,1705,1384,1089,1026,753 \mathrm{~cm}^{-1} ;{ }^{1} \mathrm{H}$ and ${ }^{13} \mathrm{C}$ NMR data, see Table S3 and S6; HRESI-MS $m / z 541.2780[\mathrm{M}+\mathrm{Na}]^{+}$(calcd. 541.2772). 
Tubocapsanolide H (27): white powder; mp. 242-244 ${ }^{\circ} \mathrm{C} ;[\alpha]_{\mathrm{D}}^{25.8}-57.5(c 0.07, \mathrm{MeOH})$;

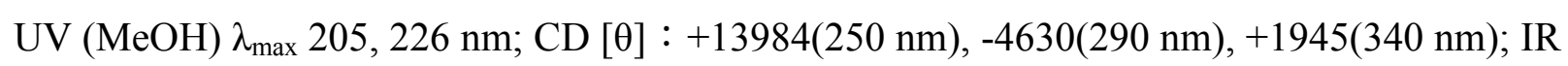
(Neat) $v_{\max } 3431,2922,2856,1705,1376,1129,1093,750 \mathrm{~cm}^{-1} ;{ }^{1} \mathrm{H}$ and ${ }^{13} \mathrm{C} \mathrm{NMR}$ data, see Table S3 and S6; HRESI-MS m/z 523.2671 [M+Na $]^{+}$(calcd. 523.2666). 